\title{
Placenta Percreta Causing Maternal Collapse due to Uterine Rupture in Third Trimester of Pregnancy: Case Report
}

\author{
Nousheen Khan*1, Sweety Kumari ${ }^{2}$, Shalini Malhotra*3 and Muna Khalfan ${ }^{4}$ \\ ${ }^{1}$ MBBS, DGO, MRCOG, MRCPI-Specialist, Al Qassimi Women and Child Health Hospital, UAE \\ ${ }^{2} M B B S, D G O, M R C O G$, Specialist, Al Qassimi Women and Child Health Hospital, UAE \\ ${ }^{3} M B B S, M D, D N B, M R C O G$, Consultant, UAE
}

${ }^{4}$ Head Of Department, Al Qassimi Women and Child Health Hospital, UAE

*Corresponding author: Nousheen Khan, MBBS, DGO, MRCOG, MRCPI-Specialist, Al Qassimi Women and Child Health Hospital, UAE

Shalini Malhotra, MBBS,MD, DNB, MRCOG, consultant, UAE

\begin{tabular}{l} 
ARTICLE INFO \\
\hline Received: 幽 August 28, 2019 \\
Published: 蔧 September 09, 2019 \\
Citation: Nousheen Khan, Sweety Ku- \\
mari, Shalini Malhotra, Muna Khalfan. \\
Placenta Percreta Causing Maternal Col- \\
lapse due to Uterine Rupture in Third \\
Trimester of Pregnancy: Case Report. \\
Biomed J Sci \& Tech Res 21(2)-2019. \\
BJSTR. MS.ID.003563.
\end{tabular}

\begin{abstract}
Spontaneous uterine rupture can lead to massive intraperitoneal bleeding which can lead to death if not recognized in time. This case recounts a 34-year-old gravida 2 term 1 live 1(G6P1001) at 33 gestational age (GA) with a past medical history of one previous $\mathrm{C}$-sections, and low-lying placenta who presented to the emergency department (ED) in a state of hypovolemic shock. Clinical findings suspected uterine rupture. The patient underwent an emergency C-section at 33 weeks GA as well as a peripartum subtotal hysterectomy in view of placenta percreta and uncontrolled PPH. During the operation, the patient suffered a postpartum hemorrhage (PPH) of $4000 \mathrm{~mL}$. She was later discharged home on postoperative day (POD) eight.
\end{abstract}

Keywords: Maternal Collapse; Shock; Peripartum; Fetal Heart Rate Tracing; Uterine Rupture; Placenta Percreta; Spectrum; Postpartum Hemorrhage

Abbreviations: GA: Gestational Age; ED: Emergency Department; PPH: Postpartum Hemorrhage; POD: Postoperative Day

\section{Introduction}

Spontaneous rupture of the non-labouring uterus leading to maternal collapse is a relatively rare occurrence in obstetrics. Although cases reported in the literature have described this event in unscarred and primigravid uterus; there have been strong associations with abnormal placentation, previous uterine surgery and congenital abnormality of the uterus. The differential diagnosis of maternal collapse can range from hemorrhage, uterine rupture, thromboembolism, sepsis, cardiac disease, drug toxicity. However, patients with risk factors such as previous cesarean sections (c-sections) and low-lying placenta, are at greater risk of developing complications earlier than we would traditionally expect [1-5].

\section{Case Report}

This case describes an unbooked 34-year-old G2P1 at 33 weeks GA who was brought to ED by ambulance, with complaint of severe backache, abdominal pain, sweating and not able to stand since 30 minutes. Her past medical history was significant for placenta previa and gestational diabetes in current pregnancy and previous 1 Cesarean section. In the ambulance, she was found to be sweaty, pale, her saturation oxygen was 62 percent, blood pressure $74 / 47 \mathrm{~mm} \mathrm{Hg}$. After arrival, she was taken to the red zone for simultaneous evaluation and stabilization by emergency team and senior obstetrician. Consultant obstetrician who was on floor also 
attended the patient immediately. On arrival, she was disoriented, anxious, groaning in pain and dyspneic. On examination, she was pale, clammy, she was unable to lie on her back as she had severe pain in the upper abdomen and back. Per abdominally, her uterus was 34 weeks with generalized tenderness, no scar pain, no contractions, Speculum examination showed closed os with no bleeding [6-9].

Bedside ultrasound was done fetus found to have bradycardia up to 70 beats/minute, placental lakes could be seen. Her vitals continued to detoriate, spo 2 still at 72 percent, pulse $114 / \mathrm{min}$, blood pressure-84/56mm Hg. Because of suspected uterine rupture, urgent Cesarean section was performed under endotracheal anesthesia. Blood bank was alerted regarding need for blood products in view of maternal shock. Laparotomy revealed that uterus was ruptured about $3 \mathrm{~cm}$ just on right side above previous scar with placenta invading the uterus wall and bladder in the lower segment more on right side, there was hemoperitoneum of 1500 liters ,intra operative diagnosis was rupture uterus with placenta percreta. Urologist was summoned to attend immediately. The ruptured opening was expanded by fingers to deliver an alive baby _ with 0/1 Apgar who was immediately intubated and shifted to NICU. Placenta was found to cover whole lower segment and cervix .Placenta and membrane could not be separated as it was reaching up to the bladder [10].

There was abundant bleeding from the placental site and atonic uterus. Uterotonics were prescribed, carbetocin $100 \mathrm{mg}$ iv single dose/ oxytocin 40 units drip inj haemabate 250 ugm IM and intra myometrium at interval of $20 \mathrm{mins} /$ inj (repeated twice) cyclokapron $1 \mathrm{gm}$ iv 2 doses at interval of 1 hour ,misoprostol 800 ugm per rectally, Bladder was dissected down by the urologist. Bilateral uterine artery ligation was done. Placental bed suture was applied to control bleeding, but patient was still hypotensive with atonic uterus. Because of placenta percreta, atonic uterus and failed 1st and 2nd line management of PPH, subtotal cesarean hysterectomy was performed with conservation of both tubes and ovaries. Total blood loss was 4 liters. Patient received 5 units of blood /4 units of FFP/4 units of platelets and three units of cryoprecipitate and calcium gluconate intraoperatively. Bladder integrity was confirmed by methylene blue and intraperitoneal drain was inserted, patient shifted to high dependency unit for closed monitoring the operating time was 2 hours and 30 minutes. Post operatively received 2 additional units of blood along with 2 units of fresh frozen plasma. She was started on intravenous ceftriaxone and metronidazole [11-12].

She made early recovery, was extubated in the 6 hours, her vitals and repeat laboratory results were within reference values, she was encouraged early mobilization, and moved out to the Postnatal ward on day 3 , she was mobilizing well, her drain was removed on day 2 after minimal collection. She was explained about manual milk expression from day 1 for feeding the baby, and was encouraged breast feeding as baby also was stable in the NICU, and had good sucking reflexes. Patient had 2 episodes of fever and chills from day 4, responded to routine analgesics, white blood cell counts 11.63 , her blood cultures were normal but high vaginal swab and urine culture showed heavy growth of E.coli. She received antibiotics for 7 days total with thromboprophylaxis and was discharged on day 8 in stable condition. Husband and patient were regularly debriefed by the attending obstetric team and offered counseling services to help cope with turnoff events [13-14].

\section{Discussion}

Spontaneous uterine rupture due to placenta percreta is a rare obstetric emergency with an estimated incidence of $1 / 5,000$ pregnancies [15]. Placenta percreta accounts for $5 \%$ of all placental invasion anomalies [16]. The main risk factor is previous cesarean operation $[13,17]$. The majority of uterine ruptures occur during the third trimester due to thinned uterine lower segment .Similar case in Saudi Medical Journal 14. Fortunately, with identification of maternal shock, presence of multidisciplinary team with senior involvement, early identification of placenta accreta and early resort to hysterectomy in view of massive hemorrhage, patient's life was saved. This was a very important event in the couple's life and very important to identify debriefing of not only the patient but also to the husband in view of the untoward incident. The number of births by cesarean section is on the rise, climbing from about 16 million (12.1\% of all births) in 2000 to 29.7 million (21.1\% of all births) in 2015, according to a study in the journal The Lancet., Women with prior CS are at higher risk of uterine rupture. The reported incidence of uterine rupture among women with prior CS ranged from $0.22 \%$ to $0.5 \%$. The strategies for prevention and management, as well as the quality of affordable care for women at risk of or experiencing uterine rupture, are likely to vary across settings depending on their diagnostic capacity, availability of obstetric interventions, and human and facility resources.

Factors that potentially increase the risk of uterine rupture include short inter-delivery interval (less than 12 months since last delivery), post-date pregnancy, maternal age of 40 years or more, and obesity, lower prelabour Bishop Score, macrosomia and decreased ultrasonographic lower segment myometrial thickness. However, no safety studies on appropriate interval after cesarean section to conceive. Met analysis has shown that hysterectomy was required in $14-33 \%$ of uterine rupture cases. All epidemiological studies of the last 2 decades have shown a direct association between the increase in caesarean deliveries and the incidence of placenta accreta spectrum (abnormally adherent and invasive placenta) in subsequent pregnancies worldwide. The 2016 Nordic Obstetric Surveillance Study found that the risk of invasive placentation increases seven-fold after one prior caesarean section. Maternal complications in placenta accreta spectrum are primarily 
the result of massive haemorrhage. Median estimated blood loss in cohorts of placenta accreta spectrum ranges from 2000 to $7800 \mathrm{ml}$ and the median number of units of blood transfused is 5 units.

Antenatal diagnosis of placenta accreta spectrum reduces maternal peripartum hemorrhage and morbidity. Multidisciplinary management in a maternity unit with access to maternal and neonatal intensive care is often required for women with placenta accreta spectrum. For such care to be organized, the diagnosis must be made antenatally. Overall, women with percreta placentation should be cared for according to the risks of severe maternal bleeding and premature delivery. Placenta percreta can be associated with major prenatal complications from early in pregnancy, such as uterine rupture and bladder involvement with associated lifethreatening haemorrhage in cases of suspected placenta accreta spectrum, where significant blood loss and caesarean hysterectomy is anticipated, delivery at between 34 and 35 weeks of gestation has been proposed in order to avoid emergency delivery, which still occurs about $20 \%$ of the time even in scheduled cases. A 2010 decision analysis supports this approach based on the increasing likelihood of emergency delivery as pregnancy goes beyond 34 weeks of gestation. The American College of Obstetricians and Gynecologists (ACOG) guidelines highlight that to enhance patient safety, it is important that the delivery be performed by an experienced obstetric team that includes an obstetric surgeon, with other surgical specialists, such as urologists, general surgeons, and gynecological surgeons and oncologists, available if necessary.

\section{References}

1. Vahanian SA, Lavery JA, Ananth CV, Vintzileos A (2015) Placental implantation abnormalities and risk of preterm delivery: a systematic review and metaanalysis. Am J Obstet Gynecol 213(S4): 78-90.

2. Wu S, Kocherginsky M, Hibbard JU (2005) Abnormal placentation: twenty-year analysis. Am J Obstet Gynecol 192: 1458-1461.

3. Farquhar CM, Li Z, Lensen S, McLintock C, Pollock W, et al. (2017) Incidence, risk factors and perinatal outcomes for placenta accreta in Australia and New Zealand: a casecontrol study. BMJ Open 7(10): e017713.

\section{ISSN: 2574-1241}

DOI: 10.26717/BJSTR.2019.21.003563

Nousheen Khan, Shalini Malhotra. Biomed J Sci \& Tech Res

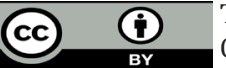

This work is licensed under Creative Commons Attribution 4.0 License

Submission Link: https://biomedres.us/submit-manuscript.php
4. Wright JD, Pri Paz S, Herzog TJ, Shah M, Bonanno C, et al. (2011) Predictors of massive blood loss in women with placenta accreta. Am J Obstet Gynecol 205: 38.e1-6.

5. Shamshirsaz AA, Fox KA, Salmanian B, Diaz Arrastia CR, Lee W, et al. (2015) Maternal morbidity in patients with morbidly adherent placenta treated with and without a standardized multidisciplinary approach. Am J Obstet Gynecol 212(2): 218.e1-9.

6. Shamshirsaz AA, Fox KA, Erfani H, Clark SL, Salmanian B, et al. (2017) Multidisciplinary team learning in the management of the morbidly adherent placenta: outcome improvements over time. Am J Obstet Gynecol 216(6): 612.e1-612.

7. Silver RM, Fox KA, Barton JR, Abuhamad AZ, Simhan H, et al. (2015) Center of excellence for placenta accreta. Am J Obstet Gynecol 212(5): 561-568.

8. Smulian JC, Pascual AL, Hesham H, Qureshey E, Bijoy Thomas M, et al. (2017) Invasive placental disease: the impact of a multidisciplinary team approach to management. J Matern Fetal Neonatal Med 30(12):14231427.

9. Hornemann A, Bohlmann MK, Diedrich K, Kavallaris A, Kehl S, et al. (2011) Spontaneous uterine rupture at the $21^{\text {st }}$ week of gestation caused by placenta percreta. Arch Gynecol Obstet 284(4): 875-878.

10. Sun JN, Zhang BL, Yu HY, Zhang Q (2016) Spontaneous uterine rupture due to placenta percreta during pregnancy. Am J Emerg Med 34(9): 1918.e1-3.

11. Publications Committee, Society for Maternal Fetal Medicine, Belfort MA (2010) Placenta accreta. Am J Obstet Gynecol 203(5): 430-439.

12. (2012) Committee on Obstetric Practice. Committee opinion no. 529: placenta accreta. Obstet Gynecol 120(1): 207-211.

13. Priya Dahiya, Kanad D Nayar, Amar JS Gulati, Kiran Dahiya (2012) Placenta Accreta Causing Uterine Rupture in Second Trimester of Pregnancy after in vitro Fertilization. A Case Report J Reprod Infertil 13(1): 61-63.

14. Burcu A. Ulkumen, Halil G Pala, Yesim Baytur (2014) Acute abdomen and massive hemorrhage due to placenta percreta leading to spontaneous uterine rupture in the second trimester. Saudi Med J 35(9): 1131-1132.

15. Gardeil F, Daly S, Turner MJ (1994) Uterine rupture in pregnancy reviewed. Eur J Obstet Gynecol Reprod Biol 56(2): 107-110.

16. Roeters AE, Oudijk MA, Heydanus R, Bruinse HW (2007) Pregnancy outcome after intra-abdominal bleeding due to placenta percreta at 14 weeks of gestation. Obstet Gynecol 109(2 Pt2): 574-576.

17. Morgan K, Arulkumaran S (2003) Antepartum haemorrhage. Curr Obstet Gynecol 13(2): 81-87.

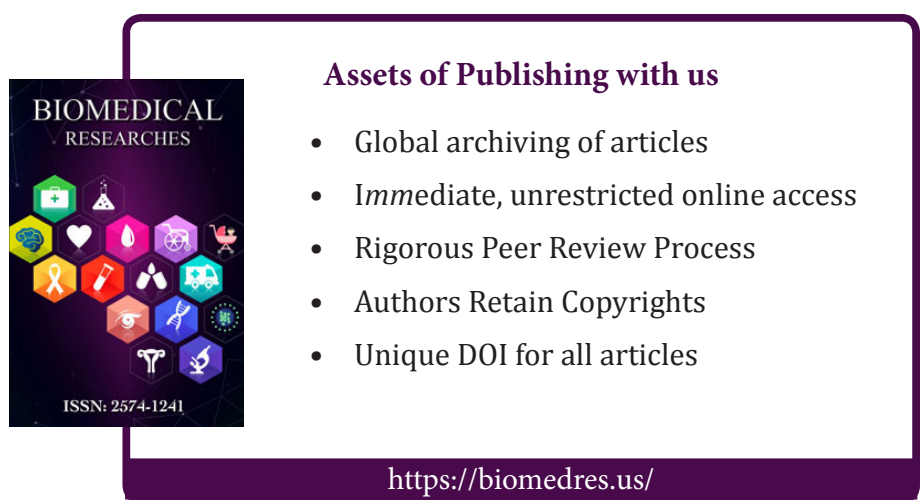

